\title{
Accélérer la cicatrisation des plaies chroniques grâce à des pansements à base de graphène : la start-up GRAPHEAL
}

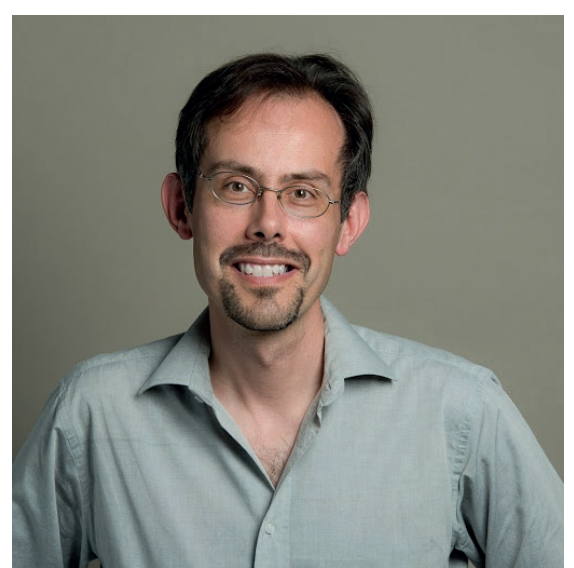

Vincent Bouchiat

\author{
La start-up grenobloise GRAPHEAL a mis au point un pansement \\ connecté, qui permet de suivre l'état des plaies chroniques en continu \\ et favorise leur cicatrisation. Vincent Bouchiat, fondateur de GRAPHEAL, \\ est interviewé par Michel Belakhovsky pour Reflets de la physique.
}

La start-up GRAPHEAL en quelques chiffres

Date de création : avril 2019

Président : Vincent Bouchiat

Nombre de collaborateurs : 5

Capital social : 50000 euros

Adresse : 25 avenue des Martyrs, 38000 Grenoble

Site internet : www.grapheal.com/

Contact : info@grapheal.fr

GRAPHEAL a été lauréate en 2019 des Innotrophées, prix organisé par la Chambre de commerce et d'industrie de Grenoble, dans la catégorie "startup ".

Elle a également remporté le troisième prix du concours national EDF Pulse pour son innovation.
Michel Belakhovsky : Vincent Bouchiat, vous étiez directeur de recherche au CNRS à I'Institut Néel de Grenoble. Vous venez de quitter provisoirement ce poste pour diriger la start-up GRAPHEAL que vous avez fondée. Je souhaiterais que vous me parliez d'abord de votre activité de recherche.

Vincent Bouchiat: J'ai travaillé ces dix dernières années au sein d'une équipe de recherche en physique des solides, spécialisée sur les propriétés tant optiques qu'électroniques de matériaux " hybrides " de basse dimension [1]. Nous nous intéressions aux propriétés nouvelles présentées par des dispositifs qui couplent des nano-objets de nature différente. Il peut s'agir d'une interaction entre une molécule et un métal ou bien entre objets organiques et inorganiques. Un cas particulièrement intéressant est celui des nano-objets conducteurs unidimensionnels comme les nanofils ou les nanotubes de carbone, ou bidimensionnels comme le graphène. Les synergies de couplage, notamment électroniques, qui en résultent, aboutissent à des propriétés étonnantes. En effet, ce qui est remarquable dans ces systèmes c'est que, du fait de leur absence de volume interne, les charges se déplacent forcément en surface et par conséquent interagissent très fortement avec leur environnement, qui peut être contrôlé par un couplage adéquat. Ce concept peut être poussé très loin, notamment pour explorer la manière dont se fait l'interface avec la matière vivante, et pour permettre d'appliquer un potentiel électrique et/ou mesurer une activité de nature électrochimique.

MB : Le contexte grenoblois n'est-il pas positif pour encourager cette synergie entre connaissances fondamentales des matériaux et applications?

VB : Il y a à Grenoble un " écosystème " très actif, grâce notamment à la proximité de l'université et du CNRS avec l'Institut Polytechnique et le CEA, dont les axes de recherche sont en grande partie basés sur la maitrise des nouveaux matériaux et leur usage dans l'industrie, notamment vers les domaines d'ingénierie de pointe tels que l'électronique et l'optique. Dans notre cas, à partir de la maitrise de la croissance du graphène, notre équipe s'est ouverte à nombre de domaines : le magnétisme, la supraconductivité, l'optique et plus récemment la biologie et la médecine. Maitriser la croissance d'un matériau aussi polyvalent que le graphène permet de se placer de fait à la croisée des chemins et d'explorer des travées insoupçonnées. 
MB : Pouvez-vous rappeler les propriétés essentielles du graphène? VB : Le graphène peut être vu comme une couche monoatomique de carbone extraite d'un cristal de graphite (fig. 1). Il n'a donc pas de volume interne mais se limite essentiellement à deux surfaces, dos à dos, totalement ouvertes sur l'extérieur, ce qui le rend idéal pour réaliser des couplages.

Le graphène possède une caractéristique rare : il est relativement facile à isoler et peut garder son intégrité physique sous la forme d'une feuille d'extension latérale macroscopique et pourtant d'épaisseur atomiquement fine [2]. Il peut aussi être manipulé et conservé dans un liquide ou bien à l'air ambiant, et il résiste à son propre poids, permettant de réaliser des membranes ultimement fines, d'extension millimétrique lorsqu'il est suspendu, ou centimétrique lorsqu'il est déposé sur un substrat. Cette propriété inattendue et très singulière est liée au fait que la liaison covalente carbonecarbone est l'une des plus stables dans la nature et que le graphène ne possède pas de liaisons chimiques "pendantes ", c'est-à-dire non satisfaites, le rendant ainsi chimiquement peu réactif.

Le graphène peut être vu comme un arrangement cristallin en nid d'abeilles d'électrons $\mathrm{sp}^{2}$, c'est-à-dire une macromolécule polyaromatique "ultralourde ». Ce matériau présente ainsi une ambivalence entre l'inorganique (domaine principal de l'électronique) et l'organique (domaine de la biologie) : il n'est composé que d'atomes de carbone, mais sur les bords et les défauts du cristal, d'autres atomes peuvent être liés, typiquement de l'hydrogène ou de l'oxygène. On peut donc l'utiliser comme une très grosse molécule organique, sur laquelle il est possible de greffer ce qu'on souhaite.

MB : Quels sont les principaux pays en pointe sur les applications du graphène?

VB : Ce matériau a été initialement isolé à Manchester il y a quinze ans. Mais aujourd'hui, le plus grand acteur sur les applications est la Chine. Ce pays a identifié assez tôt le graphène comme matériau stratégique, et cela a coïncidé avec son essor technologique. Il y avait aussi pour la Chine un intérêt idéologique, i.e. donner un aspect "high-tech" au carbone, ressource minière importante qui a grandement contribué à son accession éclair à un statut de superpuissance économique. Le charbon y a constitué, comme en Europe bien avant, la ressource-clef de sa révolution industrielle. Le sous-sol chinois étant riche en charbon, le matériau graphène rencontre un vif intérêt, notamment parce qu'il permet de relancer les recherches dans ce domaine.

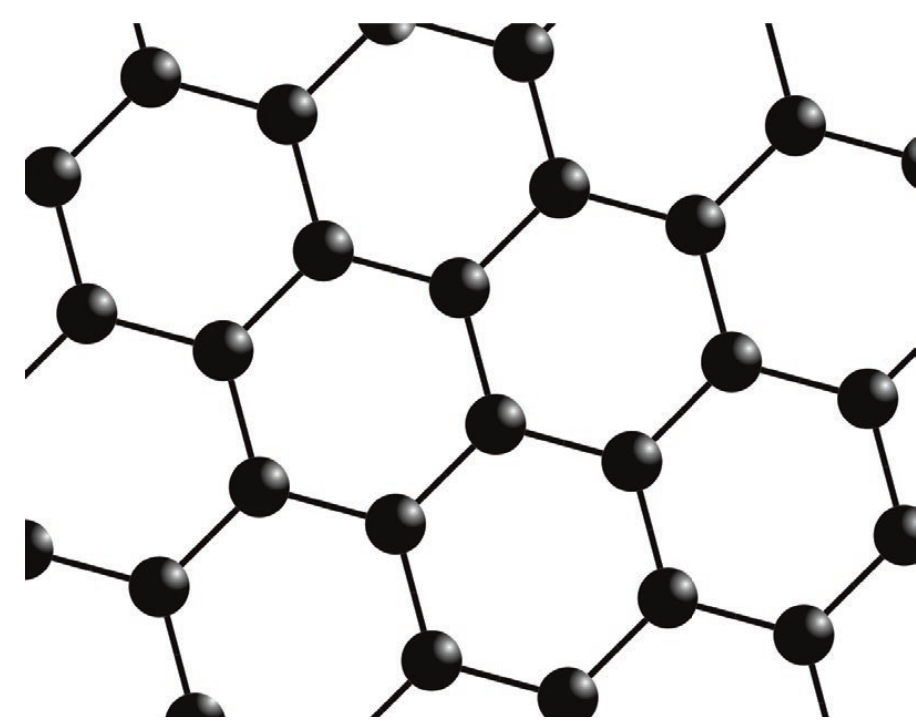

1. Représentation schématique de la structure du graphène.

MB : Avant d'aborder l'application médicale, pouvez-vous retracer I'historique de votre start-up GRAPHEAL ?

VB : GRAPHEAL est un "mot-valise ", contraction de graphène et "healing" (guérison, en anglais). Le mot introduit la finalité de notre projet industriel, à savoir utiliser le graphène comme substrat actif pour mesurer au sein de la plaie le processus de cicatrisation et la restauration du tissu cutané, réalisant ainsi la brique "hardware" d'un assistant électronique de soin des plaies difficiles à guérir.

\section{MB : Au départ, votre projet de start-up ne s'appelait-t-il pas GRAPHEAT ?}

VB : Oui, et le fait de juste changer la dernière lettre pour adapter le nom à notre application finale est une petite pirouette qui montre à quel point la valorisation est une démarche complexe qui doit se nourrir de la notion de découverte opportune, "par accident ". C'est un point qui me tient très à cœur, car il va à contre-courant de l'approche par projet qui prévaut aujourd'hui. Notre équipe avait mis au point depuis 2011 une technique de synthèse basée sur le dépôt chimique en phase vapeur du graphène et son report sur des matériaux isolants de toutes sortes - galettes de silicium, verres de lunettes, fenêtres en verre, plastique. Une de nos premières applications a été de déposer du graphène sur des substrats transparents, pour produire, par un simple effet Joule, de la chaleur uniformément sur une surface. C'est utile, par exemple pour dégivrer des fenêtres sans modifier l'homogénéité spatiale et spectrale de la lumière transmise. D'où le nom de GRAPHEAT, avec la terminaison "heat" (chaleur, en anglais).

Ce projet fut abandonné, en raison de son cout et de la très forte concurrence sur cette application. Nous nous sommes finalement tournés vers un projet plus original et prometteur - mais aussi bien plus complexe - qui consiste à utiliser le graphène sur polymère comme un substrat "bioélectronique " dans le domaine de la santé. Le changement est drastique dans l'application et dans ses marchés, mais il est basé sur une technologie sous-jacente très proche. 


\section{MB : Il vous fallait trouver une sorte de niche sur le marché ?}

VB: Exactement. C'est un point que nous, chercheurs, oublions trop souvent : pour la valorisation, surtout dans un modèle de start-up, l'important n'est pas tant l'invention elle-même que sa capacité à résoudre un problème pratique sur un marché déjà prêt à le recevoir. Une invention reste inutile pour une start-up si elle ne trouve pas son marché immédiatement. D'où l'importance d'être accompagné dans cette démarche très exploratoire, qui demande des compétences que les chercheurs n'ont pas. Il y a eu en effet un effort pour régionaliser la valorisation de la recherche sous forme de Sociétés d'accélération du transfert de technologies (SATT). Celle de Grenoble, LINKSIUM, nous a aidé à aborder de manière cohérente et crédible ce nouveau marché. Le tournant n'a pas été trop difficile, car nous travaillions déjà à l'Institut Néel sur des projets de couplage du graphène avec des éléments biologiques.

MB : Venons-en à l'objet développé par GRAPHEAL, un assistant de soin des plaies..

VB : Grâce à ses électrons circulant en surface, le graphène peut aisément servir de capteur capable de détecter des fluctuations très faibles de charges statiques adsorbées. Il va ainsi être mis à profit pour mesurer des échanges de charges à son voisinage immédiat. On peut rendre cette mesure sélective et discriminante vis-à-vis d'espèces chimiques données, en greffant sur sa surface une couche moléculaire (brin d'ADN, enzyme, anticorps...), qui ne va réagir qu'avec un composé bien déterminé. Cette fonctionnalisation va conduire, par le biais de réactions spécifiques (oxydoréduction, hybridation de l'ADN...), à des fluctuations de charge de surface, puis, par un " effet de champ » identique mais plus sensible que celui d'un transistor en silicium, à une variation de la conductance électrique du graphène. Ainsi, le graphène est un excellent matériau permettant la transduction entre la composition d'un fluide et un courant électrique. C'est donc un biocapteur flexible, transparent et peu couteux à implémenter.

Dans la plupart des applications, le graphène est obtenu par exfoliation physico-chimique du graphite, permettant de produire des encres, des solutions de graphène à très bas cout, des nanoplaquettes... Mais dans notre cas, nous nous intéressons au graphène sous une toute autre forme : en monocouche continue et stabilisée sur polymère. Nous avons donc dû développer une technologie plus couteuse et difficile à mettre en place.

On part non pas du charbon, mais du méthane $\mathrm{CH}_{4}$ qui va se décomposer et déposer les atomes de carbone sous forme de monocouche atomique sur une structure cristalline (une feuille de cuivre). Ensuite, nous avons mis au point une technique de report sur polymère, à grande échelle et sans intervention manuelle, de cette monocouche continue de graphène. Celle-ci est alors tenue en face arrière par un plastique souple et transparent qui permet de "présenter » une surface en contact direct sur la plaie, sans risquer un relargage de nanoparticules.
MB : Des électrodes couvertes de graphène seraient moins invasives?

VB : Oui. Antoine Bourrier a travaillé durant son doctorat sur l'enrobage par du graphène d'un réseau d'électrodes commerciales, utilisé sous la forme d'un implant intracortical. Le résultat est que la présence de graphène en surface permet de mesurer plus longtemps l'activité cérébrale d'une souris au cours du temps, justement en évitant toute réaction inflammatoire. Le graphène constitue dans ce cas une couche de "vernis " conducteur imperméable, qui évite aux ions d'interagir avec le métal. Nous avons de plus prouvé que le graphène est non seulement biocompatible, mais aussi biostimulant. À son contact, les cellules se reproduisent plus facilement et sont plus mobiles. Toutes les raisons n'en sont pas comprises, mais cela pourrait être lié à sa composition carbonée inerte, à sa souplesse qui se rapproche de celle d'une paroi cellulaire, voire même à sa conduction électrique. Au laboratoire, nous avons observé ce mécanisme sur différentes cellules : neurones en culture, cellules de peau humaine, fibroblastes in vitro, et ces résultats ont été reproduits par d'autres équipes. Cette biostimulation naturelle, un simple contact graphène-cellule, a aussi été démontrée chez l'animal. Puis on dispose de toutes les caractéristiques favorables du graphène : transparence optique, flexibilité et conductivité électronique. Toutes ces propriétés nous ont amenés à imaginer des applications rapides de ce matériau, et l'une de celles que j'ai identifiées est de l'intégrer au sein d'un pansement. Pourquoi un pansement ? Parce que c'est finalement ce qu'il y a de plus simple pour une interaction entre un système biologique ouvert - une plaie - et une électronique. D'où notre idée que la première application simple à implémenter pouvait être le pansement, même si dans le détail c'est complexe, car les types de plaies sont nombreux.

Nous avons identifié un marché important, celui des plaies dites " chroniques ", car beaucoup de plaies sont traitées de manière encore insatisfaisante par le suivi actuellement mis en place, par exemple celles des diabétiques ou des personnes âgées - de façon générale toute personne souffrant de plaies qui ne guérissent pas en quelques semaines. C'est donc le pansement appliqué sur de telles plaies chroniques qui est la cible du marché de GRAPHEAL. Nous avons aujourd'hui achevé le stade préclinique, en démontrant avec divers prototypes que le graphène est utile pour accélérer la cicatrisation de plaies, y compris chroniques, mais aussi permet de renseigner sur l'état de cette cicatrisation en faisant la mesure de paramètres vitaux importants, tels que le $\mathrm{pH}$. Se manifeste donc l'ambivalence de ce matériau, qui allie l'effet thérapeutique et l'effet diagnostique, ce dernier prenant la forme d'un suivi temporel d'une information lisible sur un simple smartphone (fig. 2). Cette dualité entre thérapie et monitoring est assez rare dans des dispositifs médicaux, qui sont pour la plupart à visée unique, soit thérapeutique, soit diagnostique.

Un autre exemple d'une telle dualité est la start-up DIABELOOP, avec un dispositif de mesure en temps réel de la glycémie, grâce à une boucle de contre-réaction sur une pompe à insuline sans intervention extérieure ; l'information traitée entraine une action - en quelque sorte un pancréas artificiel. Cette double approche, qui inclut l'apprentissage issu de l'intelligence artificielle, est amenée à se développer sur l'humain. 
MB : Envisagez-vous de vous rapprocher de l'hôpital, à l'instar de Claude Feuerstein (ancien président de l'université Joseph Fourier) qui a créé le GIN (Grenoble Institut des Neurosciences) au $\mathrm{CHU}$, situant ainsi la recherche à côté de l'hôpital ?

VB : Oui, c'est ce que nous avons commencé à faire pour les essais cliniques. Disposer d'un pansement intelligent permettrait de pouvoir renvoyer le malade chez lui plus rapidement, tout en suivant à distance son évolution.

Cette démarche s'inscrit dans une tendance actuelle de la généralisation de la télémédecine, induisant gain en qualité de vie et économies pour le système de santé. Car, pour une plaie chronique, qui ne guérit pas et demande un suivi, le patient doit en principe rester à l'hôpital. Mais si l'information (obtenue à travers un plâtre, par exemple) est transmise par smartphone directement à un centre hospitalier, ou est utilisable par un infirmier à domicile, on va pouvoir libérer des lits et le cout sera réduit pour l'assurance maladie ; ceci tout en améliorant la vie du patient qui va rentrer chez lui plus tôt, et sera moins angoissé car plus conscient de son état.

\section{MB : Pouvez-vous préciser l'état actuel de GRAPHEAL ?}

VB : Créer une start-up n'est pas simple, même si c'est « à la mode " ces temps-ci. Beaucoup de jeunes se lancent, avec des résultats parfois surprenants et enthousiasmants. C'est un modèle intéressant, prôné aujourd'hui jusque dans les hautes sphères de l'État, mais qui possède aussi ses limites. J'ai envie de m'y frotter, même si je n'en ignore pas les risques. Ce n'est pas un long fleuve tranquille, mais un fleuve très sinueux avec de nombreux méandres! Ce qui m'a motivé dans ce projet est de pousser une idée, faire sortir une technologie hors du laboratoire, et de l'accompagner jusqu'au produit final! Je pense que ma mission sera terminée lorsque l'on n'aura plus besoin, pour promouvoir le produit, de dire qu'il y a du graphène dedans, puisque l'intérêt de sa fonction prévaudra sur sa composition.

Mais le passage du matériau à son usage réussi en médecine est un véritable chemin de croix, car il faut résoudre nombre de problèmes : la fiabilité, l'innocuité, le suivi qualité, la production en masse, le cout raisonnable, l'acceptation par la chaine des clients (médecins, infirmiers, patients), qu'il faut convaincre chacun avec des arguments différents; et puis, enfin, la place du produit sur le marché face à une compétition acharnée - tout en gardant la capacité de le faire évoluer technologiquement. Pour un dispositif médical pour lequel, à juste titre, les normes deviennent de plus en plus sévères, les défis sont énormes et parfois impossibles à relever rapidement pour une jeune start-up. En ce qui concerne GRAPHEAL, nous avons achevé les études précliniques du pansement intelligent et aborderons bientôt la phase la plus délicate, à savoir les premiers essais sur l'homme.

\section{MB : Où en est le développement du pansement intelligent selon l'échelle TRL (technology readiness level)?}

VB : Pour caractériser le degré de maturité d'une invention, la NASA a défini une mesure dite "TRL", graduée de 1 à 10 , de l'état d'une technologie et de son utilité pour une application. Aujourd'hui nous en serions, selon les prototypes, au niveau 6 ou 7 . C'est une étape critique, souvent appelée la "vallée de la mort»: le matériau a montré son intérêt, la preuve du concept est acquise. La phase d'étude a bénéficié des aides du laboratoire (le département Nanosciences de l'Institut Néel) et des incubateurs.

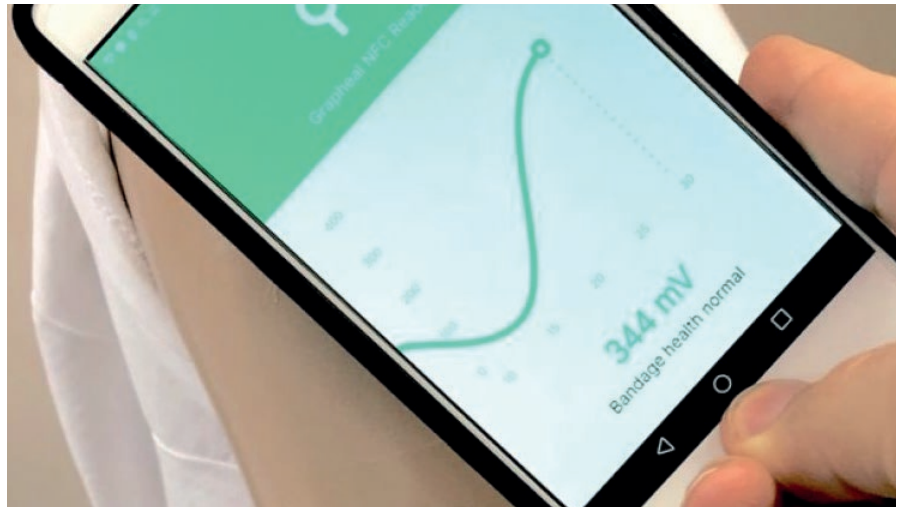

2. Principe du pansement intelligent en graphène, couplé à une lecture par smartphone et permettant d'extraire des données médicales au sein même de la plaie, sans avoir à retirer le pansement.

Pour la suite, on doit rechercher des fonds privés en montrant l'intérêt de la technologie, et ainsi la faire progresser aux TRL suivants, jusqu'au produit commercialisable. Il faut pour cela prouver au marché que le matériau et son application ont un intérêt commercial.

À ce stade, le chercheur est parfois désemparé, car la manière de mettre en valeur un produit n'est pas du tout la même quand on parle à des chercheurs ou à des investisseurs. Il faut changer de casquette, de métier, passer de chercheur appliqué à entrepreneur, c'est-à-dire être plus optimiste - quitte à passer sous silence certaines difficultés : la présentation du produit doit convaincre l'investisseur. Il faut ne plus laisser paraitre la réserve et le doute pourtant nécessaires à l'attitude du chercheur, et que le créateur de start-up doit néanmoins garder aussi en tête s'il veut réussir sur le long terme. Ce travail d'intériorisation du risque demande de la pratique...

\section{MB : Pouvez-vous préciser quels sont vos besoins en termes} de financements?

VB : Une start-up dans le biomédical demande malheureusement beaucoup d'investissements, et les dépenses sont éparpillées sur tous les fronts : faire monter en gamme le matériau, développer l'électronique et les logiciels, surveiller les aspects de qualité et de pharmacovigilance du matériau pour l'application en santé, et notamment assurer sa tenue et son innocuité, tout ceci dans le cadre réglementaire aujourd'hui plus contraignant que nécessite le dispositif médical. Il faut enfin pouvoir lever des fonds à hauteur suffisante pour financer les études cliniques. Dans le cas de la mise au point d'une molécule pour un nouveau médicament, c'est pratiquement inabordable pour une start-up, car même les grandes industries pharmaceutiques ont du mal à financer leurs recherches. Dans le cas d'un dispositif médical comme le nôtre, c'est un peu moins difficile en termes d'essais cliniques, mais il faut tout de même de l'ordre de trois ans et plus de trois millions d'euros pour lancer le produit sur le marché...

[1] Voir par exemple V. Bouchiat et W. Wernsdorfer, « Un interféromètre à nanotube de carbone ", Images de la physique, Éditions du CNRS (2009) 93-99.

[2] J.-N. Fuchs et al., " Le graphène : quand la mécanique quantique rencontre la relativité dans un trait de crayon ", Reflets de la physique, 25 (2011) 4-9. 